\title{
Finite deformations of an electroelastic circular cylindrical tube
}

\author{
Andrey Melnikov and Ray W. Ogden(D)
}

\begin{abstract}
In this paper the theory of nonlinear electroelasticity is used to examine deformations of a pressurized thick-walled circular cylindrical tube of soft dielectric material with closed ends and compliant electrodes on its curved boundaries. Expressions for the dependence of the pressure and reduced axial load on the deformation and a potential difference between, or uniform surface charge distributions on, the electrodes are obtained in respect of a general isotropic electroelastic energy function. To illustrate the behaviour of the tube, specific forms of energy functions accounting for different mechanical properties coupled with a deformation independent quadratic dependence on the electric field are used for numerical purposes, for a given potential difference and separately for a given charge distribution. Numerical dependences of the non-dimensional pressure and reduced axial load on the deformation are obtained for the considered energy functions. Results are then given for the thin-walled approximation as a limiting case of a thick-walled cylindrical tube without restriction on the energy function. The theory described herein provides a general basis for the detailed analysis of the electroelastic response of tubular dielectric elastomer actuators, which is illustrated for a fixed axial load in the absence of internal pressure and fixed internal pressure in the absence of an applied axial load.
\end{abstract}

Mathematics Subject Classification. 74B20, 74F15.

Keywords. Nonlinear electroelasticity, Electroelastic tube, Dielectric elastomer tube.

\section{Introduction}

Recent successes in the technological production of new dielectric elastomeric materials has instigated a rapid development of devices which employ the properties of such materials, including, for example, actuators and sensors, as well as noise cancelling and energy conversion devices and prototype artificial muscles. The associated nonlinear electromechanical interaction requires, in general, a rigorous continuum electromechanical theory, the development of which can be traced to the middle of the last century in the seminal work of Toupin [1], who was concerned with the theory governing elastic dielectric materials. Books dealing with the theory include [2-5]. The approach to the theory in the form described by Dorfmann and Ogden [6], however, has led to further developments and has proved to be amenable to the solution of boundary-value problems, as exemplified in [7] and the recent monograph by Dorfmann and Ogden [8] and references therein.

Included in the latter works was the analysis of the deformation of a circular cylindrical tube subject to a radial electric field with the field permeating both the interior and exterior spaces. As far as the application of the tube geometry to actuator technology is concerned a more realistic setup involves a tube with compliant electrodes coated on its curved boundaries, and this is the arrangement that is considered in the present paper. Indeed, this was one of the possible actuator geometries mentioned in $[9,10]$ in which prototype actuators were considered as proof of concept for actuating dielectric elastomers by an electric field. The corresponding problem for a spherical shell subject to internal pressure and compliant electrodes on its inner and outer surfaces was analysed in [11]. 
The boundary-value problem for a tube with closed ends considered in this paper includes both an internal pressure, which contributes to the axial load on the ends of the tube, and compliant electrodes on its inner and outer major surfaces. The actuating (axial) force can be generated by inflation and/or an electric field, and is more versatile than if the actuation is purely from an electric field since it can take advantage of both actuation mechanisms. For example, actuation by inflation can be used for the delicate handling of fragile objects where a soft touch is needed [12], while actuation by an electric field can be advantageous for some applications where rapid and accurate deformation is required [13]. The collection of papers in the volume by Carpi et al. [14] provides a source of information about the technology of dielectric actuators and their applications.

An early contribution to tubular actuator considerations was that by Carpi and De Rossi [15] who obtained experimental results for a silicone elastomer. Their mechanical analysis, however, was based on the linear theory of elasticity. In the nonlinear elasticity context a mathematical model for a thinwalled cylindrical fibre-reinforced pneumatic actuator was introduced in [13] in which a purely elastic incompressible strain-energy function was adopted, the effect of electric field being incorporated through the Maxwell stress. The fibres were taken to be inextensible and symmetrically and helically wound and the mode of actuation consisted of an inflation pressure followed by an applied voltage (potential difference), leading to radial expansion and, because of the fibre inextensibility, to axial shortening. The actuation response of a thick-walled tube of incompressible isotropic elastic material without internal pressure was examined in Zhu et al. [16] based on the so-called ideal dielectric elastomer, a description introduced in [17], for two different forms of the elastic part of the constitutive law. In this setting the actuation induced axial lengthening of the tube. Zhu et al. [16] also examined the electromechanical stability of the tube by considering the relationship between the applied voltage and the resulting (axial) actuation strain. To the authors' knowledge the two papers [13] and [16] constitute the main literature concerned with analysing tubular dielectric actuators in the nonlinear context.

The aim of the present paper is to analyse the response of a tubular actuator to the combination of a radial electric field, an internal pressure and an axial load for the fully nonlinear theory of electroelasticity developed in [6], first by considering a thick-walled tube and then its thin-walled specialization.

In Sect. 2 the main equations of the theory of incompressible isotropic electroelasticity are summarized, while in Sect. 3 the equations are specialized to those required for the considered circular cylindrical geometry. General expressions are obtained for the internal pressure in a tube with closed ends and the axial load on its ends. Next, by considering a simple specific form of energy function, explicit expressions for the pressure and axial load are obtained in terms of the deformation and the electrostatic potential (or charge) applied to the compliant electrodes.

From the formulas for a thick-walled tube numerical results which illustrate the dependence of the pressure and (reduced) axial load on the tube radius (via the azimuthal stretch on its inner boundary) and length (via the axial stretch) for different values of the applied potential or charge are provided in Sect. 4 for three different forms of the elastic part of the energy function for two different wall thicknesses (one relatively thin and one thicker) and compared with the results for the purely elastic case. It was found that there is very little difference qualitatively between the results for different tube thicknesses. Thus, it is appropriate to specialize to the thin-walled tube approximation, and this is done in Sect. 5, wherein explicit expressions for the pressure and (reduced) axial load are obtained in respect of a general electroelastic constitutive law.

A short discussion of activation is contained in Sect. 6 based on the thin-walled formulas from Sect. 5 by considering either zero internal pressure and activation at fixed axial load or zero reduced axial load at fixed internal pressure. Specific results are illustrated in respect of the neo-Hookean elastic model. Finally, some short concluding remarks are provided in Sect. 7. 


\section{Basic equations}

\subsection{Kinematics}

Consider an unloaded electrosensitive material continuum in the absence of an electric field. This configuration is referred to as the reference configuration, which is denoted by $\mathcal{B}_{\mathrm{r}}$. Material points in $\mathcal{B}_{\mathrm{r}}$ are labelled by the position vector $\mathbf{X}$. When subject to loads and/or an electric field the continuum deforms, the deformed configuration being denoted $\mathcal{B}$, wherein the material point $\mathbf{X}$ has become $\mathbf{x}$ and the deformation from $\mathcal{B}_{\mathrm{r}}$ to $\mathcal{B}$ is identified in terms of the vector deformation function $\chi$ so that $\mathbf{x}=\chi(\mathbf{X})$ for each $\mathbf{X}$ in $\mathcal{B}_{\mathrm{r}}$ (here we are not considering time dependence). The boundaries of $\mathcal{B}_{\mathrm{r}}$ and $\mathcal{B}$ are denoted by $\partial \mathcal{B}_{\mathrm{r}}$ and $\partial \mathcal{B}$, respectively.

Locally the deformation is described in terms of the deformation gradient tensor, denoted $\mathbf{F}$, which is defined by

$$
\mathbf{F}=\operatorname{Grad} \chi
$$

where Grad is the gradient operator defined with respect to $\mathbf{X}$. The associated right and left CauchyGreen deformation tensors, denoted $\mathbf{c}$ and $\mathbf{b}$ respectively, are defined by

$$
\mathbf{c}=\mathbf{F}^{\mathrm{T}} \mathbf{F}, \quad \mathbf{b}=\mathbf{F F}^{\mathrm{T}},
$$

and the quantity defined by $J=\operatorname{det} \mathbf{F}$ measures local volume changes, so that $J=1$ for an isochoric deformation, while for an incompressible material $J=1$ identically at each $\mathbf{X}$ in $\mathcal{B}_{\mathrm{r}}$.

\subsection{Equations governing an electrostatic field: Eulerian forms}

Let $\mathbf{E}$ and $\mathbf{D}$ denote the electric field and electric displacement vectors, respectively, in $\mathcal{B}$. Then, for the purely static situation in the absence of magnetic fields, free currents and free volumetric electric charges the required specializations of Maxwell's equations governing $\mathbf{E}$ and $\mathbf{D}$ are

$$
\operatorname{curl} \mathbf{E}=\mathbf{0}, \quad \operatorname{div} \mathbf{D}=0,
$$

where the operators curl and div are defined with respect to $\mathbf{x}$.

Let $\mathbf{E}^{\star}$ and $\mathbf{D}^{\star}$ denote the corresponding fields outside the material. In the case of free space, for example, they are related by $\mathbf{D}^{\star}=\varepsilon_{0} \mathbf{E}^{\star}$, where $\varepsilon_{0}$ is the permittivity of free space, and they satisfy the same equations as $\mathbf{E}$ and $\mathbf{D}$.

The field vectors have to satisfy the standard boundary conditions

$$
\mathbf{n} \times\left(\mathbf{E}^{\star}-\mathbf{E}\right)=\mathbf{0}, \quad \mathbf{n} \cdot\left(\mathbf{D}^{\star}-\mathbf{D}\right)=\sigma_{\mathrm{f}} \quad \text { on } \partial \mathcal{B},
$$

where $\mathbf{n}$ is the unit outward normal to $\partial \mathcal{B}$ and $\sigma_{\mathrm{f}}$ is the free surface charge on $\partial \mathcal{B}$ per unit area.

\subsection{Equations governing an electrostatic field: Lagrangian forms}

In order to develop a compact form of the constitutive law for an electroelastic material it was found advantageous in [6] to work in terms of Lagrangian forms of the electric field vectors. These are the pull back versions of $\mathbf{E}$ and $\mathbf{D}$ from $\mathcal{B}$ to $\mathcal{B}_{\mathrm{r}}$, denoted by $\mathbf{E}_{\mathrm{L}}$ and $\mathbf{D}_{\mathrm{L}}$ and defined by

$$
\mathbf{E}_{\mathrm{L}}=\mathbf{F}^{\mathrm{T}} \mathbf{E}, \quad \mathbf{D}_{\mathrm{L}}=J \mathbf{F}^{-1} \mathbf{D},
$$

where we recall that $J=\operatorname{det} \mathbf{F}$. The counterparts of the equations in (3) are

$$
\operatorname{Curl} \mathbf{E}_{\mathrm{L}}=\mathbf{0}, \quad \operatorname{Div} \mathbf{D}_{\mathrm{L}}=0,
$$

where the operators Curl and Div are defined with respect to $\mathbf{X}$. 
By defining $\mathbf{N}$ as the unit outward normal to $\partial \mathcal{B}_{\mathrm{r}}$ and making use of Nanson's formula $\mathbf{n} \mathrm{d} a=$ $J \mathbf{F}^{-\mathrm{T}} \mathbf{N d} A$ connecting area elements $\mathrm{d} a$ on $\partial \mathcal{B}$ and $\mathrm{d} A$ on $\partial \mathcal{B}_{\mathrm{r}}$ together with the connections (5) the boundary conditions (4) can be converted to Lagrangian form (see, for example, [8], section 4.4) as

$$
\left(\mathbf{F}^{\mathrm{T}} \mathbf{E}^{*}-\mathbf{E}_{\mathrm{L}}\right) \times \mathbf{N}=\mathbf{0}, \quad\left(J \mathbf{F}^{-1} \mathbf{D}^{*}-\mathbf{D}_{\mathrm{L}}\right) \cdot \mathbf{N}=\sigma_{\mathrm{F}} \quad \text { on } \partial \mathcal{B}_{\mathrm{r}},
$$

where $\sigma_{\mathrm{F}}$ is free surface charge density per unit area of $\partial \mathcal{B}_{\mathrm{r}}$, and it is noted that the value of $\mathbf{F}$ on $\partial \mathcal{B}_{\mathrm{r}}$ calculated from within $\mathcal{B}_{\mathrm{r}}$ is included since it is not defined outside the material.

\subsection{Constitutive equations}

In [6] two alternative forms of electroelastic constitutive law were introduced based on the notion of a total energy density as a function of $\mathbf{F}$ and either $\mathbf{E}_{\mathrm{L}}$ or $\mathbf{D}_{\mathrm{L}}$ as the independent electric variable. These were denoted $\Omega\left(\mathbf{F}, \mathbf{E}_{\mathrm{L}}\right)$ and $\Omega^{*}\left(\mathbf{F}, \mathbf{D}_{\mathrm{L}}\right)$, and either can be used in the development of the theory in general. Here we adopt just one of the formulations, that based on $\Omega^{*}\left(\mathbf{F}, \mathbf{D}_{\mathrm{L}}\right)$. This enables the total nominal stress tensor $\mathbf{T}$ and the Lagrangian electric field $\mathbf{E}_{\mathrm{L}}$ for an incompressible material to be given in compact forms by the formulas

$$
\mathbf{T}=\frac{\partial \Omega^{*}}{\partial \mathbf{F}}-p \mathbf{F}^{-1}, \quad \mathbf{E}_{\mathrm{L}}=\frac{\partial \Omega^{*}}{\partial \mathbf{D}_{\mathrm{L}}},
$$

with, by objectivity, $\Omega^{*}$ depending on $\mathbf{F}$ through $\mathbf{c}, p$ being a Lagrange multiplier associated with the incompressibility constraint

$$
\operatorname{det} \mathbf{F}=1 .
$$

Similarly to the nonlinear theory of elasticity the (total) Cauchy stress tensor, here denoted $\tau$, is a (partial) push forward of a total nominal stress: $\boldsymbol{\tau}=\mathbf{F T}$. The corresponding push forward of $\mathbf{E}_{\mathrm{L}}$ is, from (5), $\mathbf{E}=\mathbf{F}^{-\mathrm{T}} \mathbf{E}_{\mathrm{L}}$. Thus, in terms of $\Omega^{*}$,

$$
\boldsymbol{\tau}=\mathbf{F} \frac{\partial \Omega^{*}}{\partial \mathbf{F}}-p \mathbf{I}, \quad \mathbf{E}=\mathbf{F}^{-\mathrm{T}} \frac{\partial \Omega^{*}}{\partial \mathbf{D}_{\mathrm{L}}}
$$

where $\mathbf{I}$ is the identity tensor in $\mathcal{B}$.

For an incompressible isotropic electroelastic material, on which we focus in this paper, $\Omega^{*}$ is an isotropic function of $\mathbf{c}$ and $\mathbf{D}_{\mathrm{L}} \otimes \mathbf{D}_{\mathrm{L}}$ and hence may be expressed as a function of five independent invariants. The standard invariants

$$
\begin{aligned}
& I_{1}=\operatorname{tr} \mathbf{c}, \quad I_{2}=\frac{1}{2}\left[(\operatorname{tr} \mathbf{c})^{2}-\operatorname{tr}\left(\mathbf{c}^{2}\right)\right], \\
& I_{4}=\mathbf{D}_{\mathrm{L}} \cdot \mathbf{D}_{\mathrm{L}}, \quad I_{5}=\mathbf{D}_{\mathrm{L}} \cdot\left(\mathbf{c} \mathbf{D}_{\mathrm{L}}\right), \quad I_{6}=\mathbf{D}_{\mathrm{L}} \cdot\left(\mathbf{c}^{2} \mathbf{D}_{\mathrm{L}}\right),
\end{aligned}
$$

are adopted here, and we note that, by incompressibility, the invariant $I_{3}=\operatorname{det} \mathbf{c}=J^{2}=1$ is omitted.

When expanded in terms of the invariants the formulas (10) become

$$
\begin{aligned}
& \boldsymbol{\tau}=2 \Omega_{1}^{*} \mathbf{b}+2 \Omega_{2}^{*}\left(I_{1} \mathbf{b}-\mathbf{b}^{2}\right)-p \mathbf{I}+2 \Omega_{5}^{*} \mathbf{D} \otimes \mathbf{D}+2 \Omega_{6}^{*}(\mathbf{D} \otimes \mathbf{b D}+\mathbf{b D} \otimes \mathbf{D}), \\
& \mathbf{E}=2\left(\Omega_{4}^{*} \mathbf{b}^{-1}+\Omega_{5}^{*} \mathbf{I}+\Omega_{6}^{*} \mathbf{b}\right) \mathbf{D},
\end{aligned}
$$

where $\Omega_{i}^{*}$ is defined as $\partial \Omega^{*} / \partial I_{i}$ for $i=1,2,4,5,6$, and we recall that $\mathbf{b}$ is defined by $(2)_{2}$. form

In the absence of mechanical body forces the electromechanical equilibrium equation has the simple

$$
\operatorname{div} \boldsymbol{\tau}=\mathbf{0}
$$

since electric body forces can be written as the divergence of a second-order tensor and are incorporated through $\tau$, which, by virtue of angular momentum balance, is symmetric. 
Considering the deformed configuration $\mathcal{B}$, the boundary condition for the total Cauchy stress is expressible as

$$
\tau \mathbf{n}=\mathbf{t}_{\mathrm{a}}+\mathbf{t}_{\mathrm{m}}^{\star} \quad \text { on } \partial \mathcal{B}_{\mathrm{t}}
$$

where $\partial \mathcal{B}_{\mathrm{t}}$ is the part of the boundary where the mechanical traction $\mathbf{t}_{\mathrm{a}}$ is prescribed and $\mathbf{t}_{\mathrm{m}}^{\star}=\boldsymbol{\tau}_{\mathrm{m}}^{\star} \mathbf{n}$ is the load due to the Maxwell stress $\tau_{\mathrm{m}}^{\star}$, which is calculated from the fields outside $\mathcal{B}$ and defined by

$$
\boldsymbol{\tau}_{\mathrm{m}}^{\star}=\varepsilon_{0} \mathbf{E}^{\star} \otimes \mathbf{E}^{\star}-\frac{1}{2} \varepsilon_{0}\left(\mathbf{E}^{\star} \cdot \mathbf{E}^{\star}\right) \mathbf{I} .
$$

\section{Application to a thick-walled tube}

\subsection{Extension and inflation of a tube}

The theory of the previous section is now specialized for application to the problem of extension and inflation of a thick-walled circular cylindrical tube. The tube has closed ends and is subject to internal pressure, an axial load and a radial electric field generated by a potential difference between flexible electrodes coated on its inner and outer radial surfaces.

The reference geometry of the tube is described in terms of cylindrical polar coordinates $R, \Theta, Z$ according to

$$
A \leq R \leq B, \quad 0 \leq \Theta \leq 2 \pi, \quad 0 \leq Z \leq L,
$$

where $A$ and $B$ are the internal and external radii and $L$ is the length of the tube.

The circular symmetry is assumed to be maintained under extension and inflation so that the deformed configuration is described in terms of cylindrical polar coordinates $r, \theta, z$. Thus,

$$
a \leq r \leq b, \quad 0 \leq \theta \leq 2 \pi, \quad 0 \leq z \leq l,
$$

where $a, b$ and $l$ are the radii and the length of the tube in the deformed configuration.

Since the material is incompressible the resulting deformation is described by the equations

$$
r=f(R) \equiv \sqrt{a^{2}+\lambda_{z}^{-1}\left(R^{2}-A^{2}\right)}, \quad \theta=\Theta, \quad z=\lambda_{z} Z,
$$

where $\lambda_{z}$ is the uniform axial stretch. For this special form of deformation the deformation gradient $\mathbf{F}$ is purely diagonal with respect to the cylindrical polar axes, which are also the principal axes of the deformation. It is convenient to introduce the notation $\lambda=r / R$ for the azimuthal stretch. Then, by the incompressibility condition (9), the stretch in the radial direction is $\lambda_{r}=\lambda^{-1} \lambda_{z}^{-1}$.

The connections

$$
\lambda_{a}^{2} \lambda_{z}-1=\frac{R^{2}}{A^{2}}\left(\lambda^{2} \lambda_{z}-1\right)=\frac{B^{2}}{A^{2}}\left(\lambda_{b}^{2} \lambda_{z}-1\right),
$$

follow from Eq. $(20)_{1}$, the notations $\lambda_{a}$ and $\lambda_{b}$ being defined by

$$
\lambda_{a}=\frac{a}{A}, \quad \lambda_{b}=\frac{b}{B}, \quad b=f(B) .
$$

It follows from (21) that the sign of $\lambda^{2} \lambda_{z}-1$ is independent of the radius $r$. When this sign is positive, as would be the case for inflation of the tube at fixed length for example, $\lambda_{a} \geq \lambda \geq \lambda_{b}$.

Since, with respect to the chosen cylindrical polar coordinates, the matrix of the deformation gradient has the diagonal form $\operatorname{diag}\left[\lambda_{r}, \lambda, \lambda_{z}\right]$, the invariants $I_{1}$ and $I_{2}$ can be expressed as

$$
I_{1}=\lambda^{-2} \lambda_{z}^{-2}+\lambda^{2}+\lambda_{z}^{2}, \quad I_{2}=\lambda^{2} \lambda_{z}^{2}+\lambda^{-2}+\lambda_{z}^{-2} .
$$




\subsection{Electric field components and boundary conditions}

A potential difference applied to the flexible electrodes on the surfaces $r=a$ and $r=b$ is associated with equal charges of opposite signs on the two surfaces and generates a radial electric field within the material. By Gauss's Theorem there is then no radial field outside the tube. This statement is exact for an infinitely long tube, but for a tube of finite length edge effects may modify this. It is assumed here that the tube is long enough or thin enough so that edge effects can be neglected and the electric field can be considered as having only a radial component $E_{r}$, with $D_{r}$ the corresponding electric displacement component, each being independent of $\theta$ and $z$. Then Eq. $(3)_{1}$ is satisfied identically and $(3)_{2}$ yields

$$
\frac{\mathrm{d}}{\mathrm{d} r}(r D)=0
$$

where $D_{r}$ has been written $D=D(r)$. Thus, $r D$ is a constant, and hence

$$
r D(r)=a D(a)=b D(b) .
$$

The total charge on $r=a$ is now denoted by $Q$ (which may be positive or negative), and that on $r=b$ is therefore $-Q$. The free surface charge densities per unit area on $r=a$ and $r=b$ are

$$
\sigma_{\mathrm{f} a}=\frac{Q}{2 \pi a l}, \quad \sigma_{\mathrm{f} b}=-\frac{Q}{2 \pi b l} .
$$

From the boundary condition $(4)_{2}$ with $\mathbf{D}^{\star}=\mathbf{0}$ we obtain for the two boundaries

$$
D(a)=\sigma_{\mathrm{f} a}, \quad D(b)=-\sigma_{\mathrm{f} b},
$$

and hence $(25)$ yields

$$
r D(r)=\frac{Q}{2 \pi l} .
$$

In Sect. 2.4 we chose the work in terms of the energy function $\Omega^{*}\left(\mathbf{F}, \mathbf{D}_{\mathrm{L}}\right)$, where $\mathbf{D}_{\mathrm{L}}=\mathbf{F}^{-1} \mathbf{D}$, which is appropriate for the tube problem since the electric displacement has been determined explicitly, whereas the corresponding electric field has not. For the present problem $\mathbf{D}_{\mathrm{L}}$ has just a radial component, denoted $D_{\mathrm{L}}$ and given by $D_{\mathrm{L}}=\lambda_{r}^{-1} D=\lambda \lambda_{z} D$. From the definitions (12), the invariants $I_{4}, I_{5}$ and $I_{6}$ can then be expressed as

$$
I_{4}=D_{\mathrm{L}}^{2}, \quad I_{5}=\lambda^{-2} \lambda_{z}^{-2} I_{4}, \quad I_{6}=\lambda^{-4} \lambda_{z}^{-4} I_{4} .
$$

The electric field $\mathbf{E}$ is given in Eq. (14) but has only a radial component $E_{r}$, now denoted $E$ and given by

$$
E=2\left(\Omega_{4}^{*} \lambda^{2} \lambda_{z}^{2}+\Omega_{5}^{*}+\Omega_{6}^{*} \lambda^{-2} \lambda_{z}^{-2}\right) D
$$

\subsection{Stress components and boundary conditions}

The Cauchy stress components are obtained from (13), the only nonzero ones being

$$
\begin{aligned}
& \tau_{r r}=2 \Omega_{1}^{*} \lambda^{-2} \lambda_{z}^{-2}+2 \Omega_{2}^{*}\left(\lambda_{z}^{-2}+\lambda^{-2}\right)-p+2 \Omega_{5}^{*} D^{2}+4 \Omega_{6}^{*} \lambda^{-2} \lambda_{z}^{-2} D^{2}, \\
& \tau_{\theta \theta}=2 \Omega_{1}^{*} \lambda^{2}+2 \Omega_{2}^{*}\left[\lambda_{z}^{-2}+\lambda_{z}^{2} \lambda^{2}\right]-p, \\
& \tau_{z z}=2 \Omega_{1}^{*} \lambda_{z}^{2}+2 \Omega_{2}^{*}\left[\lambda^{-2}+\lambda^{2} \lambda_{z}^{2}\right]-p .
\end{aligned}
$$

Since the invariants are functions of two independent stretches $\lambda$ and $\lambda_{z}$ and $I_{4}$ it is convenient to define the reduced energy function $\omega^{*}$ by

$$
\omega^{*}\left(\lambda, \lambda_{z}, I_{4}\right)=\Omega^{*}\left(I_{1}, I_{2}, I_{4}, I_{5}, I_{6}\right),
$$

with $I_{1}, I_{2}, I_{5}, I_{6}$ specialized according to Eqs. (23) and (29). 
From Eqs. (31)-(33) we then obtain the simple formulas

$$
\tau_{\theta \theta}-\tau_{r r}=\lambda \omega_{\lambda}^{*}, \quad \tau_{z z}-\tau_{r r}=\lambda_{z} \omega_{\lambda_{z}}^{*},
$$

where $\omega_{\lambda}^{*}=\partial \omega^{*} / \partial \lambda$ and $\omega_{\lambda_{z}}^{*}=\partial \omega^{*} / \partial \lambda_{z}$. Similarly, from Eq. (30) we obtain

$$
E=2 \lambda^{2} \lambda_{z}^{2} \omega_{4}^{*} D,
$$

where $\omega_{4}^{*}=\partial \omega^{*} / \partial I_{4}$.

Since there is no field outside the tube, the Maxwell stress (17) is zero, and the only mechanical contribution to the boundary condition (16) is an applied pressure $P$ on the inner surface at $r=a$ (with zero traction on $r=b$ ). Thus,

$$
\tau_{r r}=-P \quad \text { on } r=a, \quad \tau_{r r}=0 \quad \text { on } r=b .
$$

The equilibrium equation (15) specializes to

$$
r \frac{\mathrm{d} \tau_{r r}}{\mathrm{~d} r}=\tau_{\theta \theta}-\tau_{r r}=\lambda \omega_{\lambda}^{*}
$$

in which $(35)_{1}$ has been used. Integration of (38) with the boundary conditions (37) yields

$$
P=\int_{a}^{b} \lambda \omega_{\lambda}^{*} \frac{\mathrm{d} r}{r} .
$$

Since $b=f(B)=\sqrt{a^{2}+\lambda_{z}^{-1}\left(B^{2}-A^{2}\right)}$ this provides an expression for the pressure $P$ in terms of the inner radius $a$ and the electric field through the invariant $I_{4}$ for any given initial geometry and axial stretch.

In addition to the internal pressure an axial load is applied to the ends of the tube, which is assumed to have closed ends so that the axial load includes a contribution from the pressure on the ends of the tube. This is denoted by $N$ and given by

$$
N=2 \pi \int_{a}^{b} \tau_{z z} r \mathrm{~d} r
$$

On use of Eqs. (35) and (38) the axial stress $\tau_{z z}$ can be expressed as

$$
\tau_{z z}=\frac{1}{2}\left[\frac{1}{r} \frac{\mathrm{d}}{\mathrm{d} r}\left(r^{2} \tau_{r r}\right)-\lambda \omega_{\lambda}^{*}\right]+\lambda_{z} \omega_{\lambda_{z}}^{*},
$$

and hence, on integration of the first term and application of the boundary conditions (37), we obtain

$$
F \equiv N-\pi a^{2} P=\pi \int_{a}^{b}\left(2 \lambda_{z} \omega_{\lambda_{z}}^{*}-\lambda \omega_{\lambda}^{*}\right) r \mathrm{~d} r,
$$

wherein the quantity $F$ is defined. This is referred to as the reduced axial load because it removes the contribution of the pressure load on the ends of the cylinder from the total axial load $N$.

3.3.1. A special material model. The theory of the previous section is completely general for an isotropic electroelastic material and admits many possible specializations. For definiteness we now consider a simple model for which the electric contribution to above formulas for $P$ and $F$ is based on the linear specialization $E=\varepsilon^{-1} D$ of the constitutive law (30), corresponding to $\Omega_{4}^{*}=\Omega_{6}^{*}=0$ and $\Omega_{5}^{*}=1 /(2 \varepsilon)$, where $\varepsilon$ is the permittivity of tube material, taken to be a constant, so that $\omega_{4}^{*}=1 /\left(2 \varepsilon \lambda^{2} \lambda_{z}^{2}\right)$, and the contribution of the electric field to $\omega^{*}$ is $\varepsilon E^{2} / 2=D^{2} /(2 \varepsilon)$. We then assume that $\omega^{*}$ has the form

$$
\omega^{*}=\omega\left(\lambda, \lambda_{z}\right)+\frac{1}{2} \varepsilon^{-1} \lambda^{-2} \lambda_{z}^{-2} I_{4},
$$


where $\omega\left(\lambda, \lambda_{z}\right)$ is a purely (isotropic) elastic contribution to the total energy, as yet remaining completely general. It follows that

$$
\lambda \omega_{\lambda}^{*}=\lambda \omega_{\lambda}-\varepsilon^{-1} \lambda^{-2} \lambda_{z}^{-2} I_{4}, \quad \lambda_{z} \omega_{\lambda_{z}}^{*}=\lambda_{z} \omega_{\lambda_{z}}-\varepsilon^{-1} \lambda^{-2} \lambda_{z}^{-2} I_{4} .
$$

Integration of the electric term in (39) and (42) leads to

$$
P=\int_{\lambda_{b}}^{\lambda_{a}}\left(\lambda^{2} \lambda_{z}-1\right)^{-1} \omega_{\lambda} \mathrm{d} \lambda-\frac{q A^{4}\left(\eta^{2}-1\right)}{2 \varepsilon \lambda_{z}^{3} a^{2} b^{2}}
$$

and

$$
F=\pi A^{2}\left(\lambda_{a}^{2} \lambda_{z}-1\right) \int_{\lambda_{b}}^{\lambda_{a}}\left(\lambda^{2} \lambda_{z}-1\right)^{-2}\left(2 \lambda_{z} \omega_{\lambda_{z}}-\lambda \omega_{\lambda}\right) \lambda \mathrm{d} \lambda-\frac{\pi q A^{2}}{\varepsilon \lambda_{z}^{2}} \log \frac{b}{a},
$$

where $\eta=B / A$ and $q$, which is independent of the deformation, is defined as

$$
q=\left(\frac{Q}{2 \pi A L}\right)^{2}
$$

and the integration variable in the remaining integrals has been changed to $\lambda$ using the connection

$$
r \frac{\mathrm{d} \lambda}{\mathrm{d} r}=-\lambda\left(\lambda^{2} \lambda_{z}-1\right),
$$

which can be obtained from the definition $\lambda=r / R$ and Eq. $(20)_{1}$.

\subsection{Charge or potential}

Since curl $\mathbf{E}=\mathbf{0}$, there exists a scalar field $\phi$ (the electrostatic potential) such that $\mathbf{E}=-\operatorname{grad} \phi$. For the present problem $\phi$ depends only on $r$, and therefore $E_{r}=E=-\mathrm{d} \phi / \mathrm{d} r$, and hence, from (36), we have

$$
\frac{\mathrm{d} \phi}{\mathrm{d} r}=-2 \lambda^{2} \lambda_{z}^{2} \omega_{4}^{*} D
$$

Integration of this using (28) gives the potential difference between the surfaces as

$$
\phi(b)-\phi(a)=-\frac{Q}{\pi l} \lambda_{z}^{2} \int_{a}^{b} \lambda^{2} \omega_{4}^{*} \frac{\mathrm{d} r}{r} .
$$

For the model (43) this yields the explicit formula

$$
\phi(b)-\phi(a)=-\frac{Q}{2 \pi l \varepsilon} \log (b / a),
$$

which provides a relationship between the potential difference between the inner and outer surfaces, the charge $Q$, the inner radius $a$ and the length of the cylinder $l$. Note that in terms of the reference coordinate $R$ the potential may be written as $\Phi(R)=\phi(r)$, so that the potential difference can also be written as $\Phi(B)-\Phi(A)$.

We now introduce the notation $E_{0}$, which is the mean value of the potential through the undeformed thickness, i.e.,

$$
E_{0}=\frac{\phi(b)-\phi(a)}{B-A} .
$$

This is a convenient measure of the potential difference, and is connected to the charge through $q$ by

$$
\varepsilon E_{0}^{2}=\frac{q[\log (b / a)]^{2}}{\lambda_{z}^{2}(\eta-1)^{2} \varepsilon},
$$


where, we recall, $\eta=B / A$. This allows the formulas (45) and (46) to be expressed in terms of the potential difference as

$$
P=\int_{\lambda_{b}}^{\lambda_{a}}\left(\lambda^{2} \lambda_{z}-1\right)^{-1} \omega_{\lambda} \mathrm{d} \lambda-\frac{\varepsilon E_{0}^{2}\left(\eta^{2}-1\right)(\eta-1)^{2}}{2 \lambda_{z} \lambda_{a}^{2} \lambda_{b}^{2} \eta^{2}[\log (b / a)]^{2}}
$$

and

$$
F=\pi A^{2}\left(\lambda_{a}^{2} \lambda_{z}-1\right) \int_{\lambda_{b}}^{\lambda_{a}}\left(\lambda^{2} \lambda_{z}-1\right)^{-2}\left(2 \lambda_{z} \omega_{\lambda_{z}}-\lambda \omega_{\lambda}\right) \lambda \mathrm{d} \lambda-\pi A^{2} \frac{\varepsilon E_{0}^{2}(\eta-1)^{2}}{\log (b / a)} .
$$

To be more specific we now consider a simple energy function for which the purely elastic part corresponds to the neo-Hookean model, so that

$$
\Omega^{*}\left(I_{1}, I_{5}\right)=\frac{1}{2} \mu\left(I_{1}-3\right)+\frac{1}{2} \varepsilon^{-1} I_{5},
$$

where the constant $\mu$ is the shear modulus of the neo-Hookean material in the absence of an electric field. It follows that

$$
\omega^{*}=\omega+\frac{1}{2} \varepsilon^{-1} \lambda^{-2} \lambda_{z}^{-2} I_{4}, \quad \omega=\frac{1}{2} \mu\left(\lambda^{2}+\lambda_{z}^{2}+\lambda^{-2} \lambda_{z}^{-2}-3\right),
$$

and hence

$$
\lambda \omega_{\lambda}=\mu\left(\lambda^{2}-\lambda^{-2} \lambda_{z}^{-2}\right), \quad \lambda_{z} \omega_{\lambda_{z}}=\mu\left(\lambda_{z}^{2}-\lambda^{-2} \lambda_{z}^{-2}\right),
$$

and the integrals in (45) and (46) can be evaluated explicitly to give

$$
P=\mu\left[\lambda_{z}^{-1} \ln \left(\lambda_{a} / \lambda_{b}\right)+\lambda_{z}^{-2} \frac{\lambda_{a}^{2}-\lambda_{b}^{2}}{2 \lambda_{b}^{2} \lambda_{a}^{2}}\right]-\frac{q A^{4}\left(\eta^{2}-1\right)}{2 \varepsilon \lambda_{z}^{3} a^{2} b^{2}}
$$

and

$$
F=\pi A^{2} \mu\left[\left(\lambda_{z}-\lambda_{z}^{-2}\right)\left(\eta^{2}-1\right)-\lambda_{z}^{-2}\left(\lambda_{a}^{2} \lambda_{z}-1\right) \log \left(\lambda_{a} / \lambda_{b}\right)\right]-\frac{\pi q A^{2}}{\varepsilon \lambda_{z}^{2}} \log (b / a) .
$$

The formulas (59) and (60) give expressions for $P$ and $F$ in terms of $\lambda_{a}$ and $\lambda_{z}$ and, via $q$, the charge, and again we recall that $\lambda_{b}$ can be expressed in terms of $\lambda_{a}$ and $\lambda_{z}$.

The formula (59) for $P$ may also be written in terms of the potential difference as

$$
P=\mu\left[\lambda_{z}^{-1} \ln \frac{\lambda_{a}}{\lambda_{b}}+\lambda_{z}^{-2} \frac{\lambda_{a}^{2}-\lambda_{b}^{2}}{2 \lambda_{b}^{2} \lambda_{a}^{2}}\right]-\frac{\varepsilon E_{0}^{2}\left(\eta^{2}-1\right)(\eta-1)^{2}}{2 \lambda_{z} \lambda_{a}^{2} \lambda_{b}^{2} \eta^{2}[\log (b / a)]^{2}}
$$

and likewise

$$
F=\pi A^{2}\left[\left(\lambda_{z}-\lambda_{z}^{-2}\right)\left(\eta^{2}-1\right)-\lambda_{z}^{-2}\left(\lambda_{a}^{2} \lambda_{z}-1\right) \log \left(\lambda_{a} / \lambda_{b}\right)\right]-\pi A^{2} \frac{\varepsilon E_{0}^{2}(\eta-1)^{2}}{\log (b / a)} .
$$

Note that for $P=0$ elimination of either $q$ from (59) and (60) or $E_{0}$ from (61) and (62) yields a formula for $F$ which is equivalent to one obtained in [16] but expressed in different notation.

\section{Numerical results}

In this section, in dimensionless form with $P^{*}=P / \mu$ and $F^{*}=F /\left(\pi \mu A^{2}\right)$, we illustrate the dependence of $P$ and $F$ on $\lambda_{a}$ based on Eqs. (45) and (46) for different fixed (dimensionless) values of $q$, defined by $q^{*}=q /(\mu \varepsilon)$, and Eqs. (54) and (55) for different fixed (dimensionless) values of $E_{0}^{2}$, defined by $e^{*}=\varepsilon E_{0}^{2} / \mu$, 
with $\lambda_{z}=1.2$ in each case and for three different forms of the elastic energy function $\omega\left(\lambda, \lambda_{z}\right)$. The same shear modulus $\mu$ is used for each of the three models. These are, first, the neo-Hookean model

$$
\omega\left(\lambda, \lambda_{z}\right)=\frac{1}{2} \mu\left(\lambda^{2}+\lambda_{z}^{2}+\lambda^{-2} \lambda_{z}^{-2}-3\right),
$$

and second, the Ogden model [18] for which

$$
\omega\left(\lambda, \lambda_{z}\right)=\sum_{n=1}^{3} \frac{\mu_{n}}{\alpha_{n}}\left(\lambda^{\alpha_{n}}+\lambda_{z}^{\alpha_{n}}+\lambda_{z}^{-\alpha_{n}} \lambda^{-\alpha_{n}}-3\right),
$$

where $\mu_{n}$ and $\alpha_{n}, n=1,2,3$, are material constants and the shear modulus $\mu$ is given by

$$
2 \mu=\sum_{n=1}^{3} \mu_{n} \alpha_{n}
$$

Specifically, we use the following values of the material constants, with $\mu_{n}^{*}=\mu_{n} / \mu$, which were obtained by fitting data for a vulcanized natural rubber:

$$
\alpha_{1}=1.3, \quad \alpha_{2}=5.0, \quad \alpha_{3}=-2.0, \quad \mu_{1}^{*}=1.491, \quad \mu_{2}^{*}=0.0028, \quad \mu_{3}^{*}=-0.0237 .
$$

The third model is the limiting chain extensibility model of Gent [19] for which

$$
\omega\left(\lambda, \lambda_{z}\right)=-\frac{\mu G}{2} \log \left[1-\frac{\left(\lambda^{2}+\lambda_{z}^{2}+\lambda^{-2} \lambda_{z}^{-2}-3\right)}{G}\right],
$$

where $G$ is a dimensionless material constant, which is set here to the value $G=97.2$ obtained by Gent in fitting data for vulcanized rubber.

Figures 1 and 2 are for $\eta=B / A=1.1$, a relatively thin-walled tube, while Figs. 3 and 4 are for a relatively thick-walled tube with $\eta=1.5$. Mathematica [20] is used for the numerical calculations.

In Fig. $1 P^{*}$ is plotted against $\lambda_{a}$. In each panel of the left-hand column four different fixed values of $q^{*}$ are used while for each panel in the right-hand column results are shown for four different fixed values of $e^{*}$. The first, second and third rows, respectively, correspond to the neo-Hookean, Ogden and Gent models.

These results (with $\eta=1.1$ ) are, not surprisingly, consistent with the explicit expressions for $P$ and $F$ for a thin-walled tube to be presented in Sect. 5. Indeed, calculations for thinner walled tubes (e.g., $\eta=1.01$ ) reveal the same qualitative features as for $\eta=1.1$. In particular, for fixed charge the influence of the electric contribution declines with increasing azimuthal stretch $\lambda_{a}$ whereas an increasing potential difference has a progressively negative influence for all $\lambda_{a}$ for each of the models.

It is well known from rubber elasticity that the range of deformations for which the neo-Hookean model is applicable is limited. This is reflected in the plots in Fig. 1a, b where there is an upper limit to the pressure that can be sustained with $P$ tending to an asymptote as $\lambda_{a}$ becomes larger and larger. The upper curve corresponds to the purely elastic case. The asymptote is the same for all values of $q^{*}$ in Fig. 1a but reduces in Fig. 1b with increasing $e^{*}$. For any pressure (including $P^{*}=0$ ) below the asymptotic value the effect of an applied $q^{*}$ is to increase $\lambda_{a}$, i.e., to increase the radius. This effect is the same, but somewhat less pronounced, for an applied $e^{*}$.

In the case of Fig. 1c, d there is no upper limit to the pressure and, for any given pressure, an increase in $q^{*}$ or $e^{*}$ has the effect of increasing the radius. For this model, however, there is a maximum in the pressure followed by a minimum and subsequent monotonic increase as $\lambda_{a}$ increases. This phenomenon is well known for rubber balloons, is associated with snap through instability and is maintained in the presence of an electric field for a dielectric elastomer balloon (see [21] for the case of a spherical balloon). In Fig. 1d the pressure required to achieve a given inflation reduces as the applied potential difference increases, to the point that (illustrated for $e^{*}=0.55$ ) snap though instability can occur in the absence of pressure. 


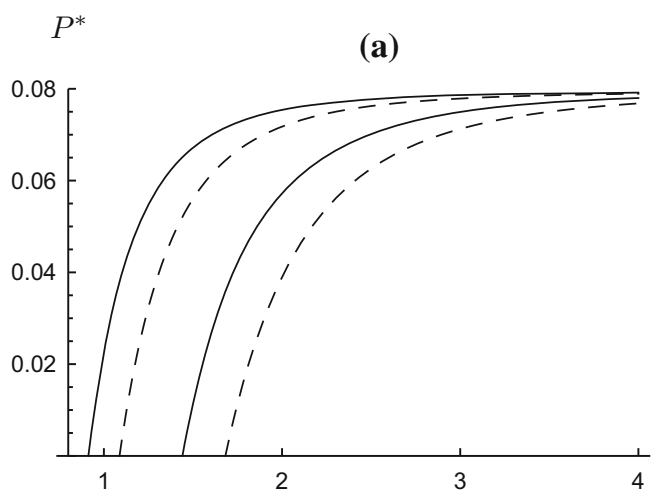

(c)

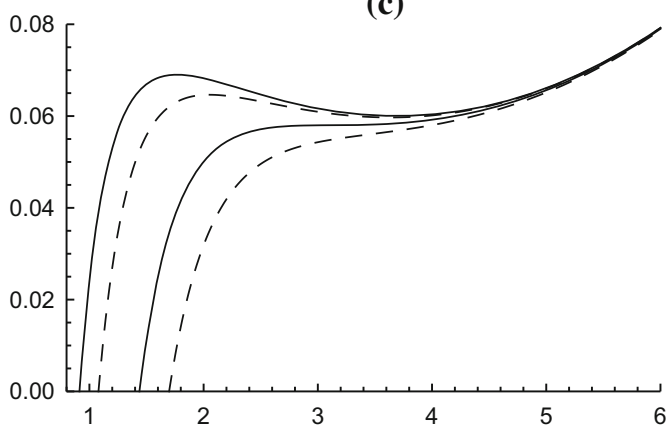

(e)

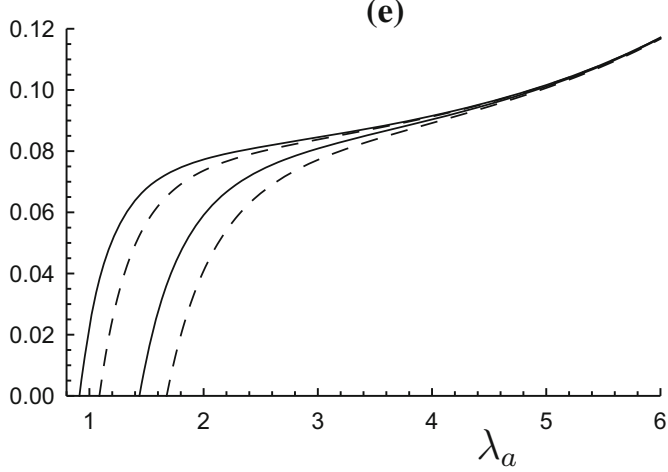

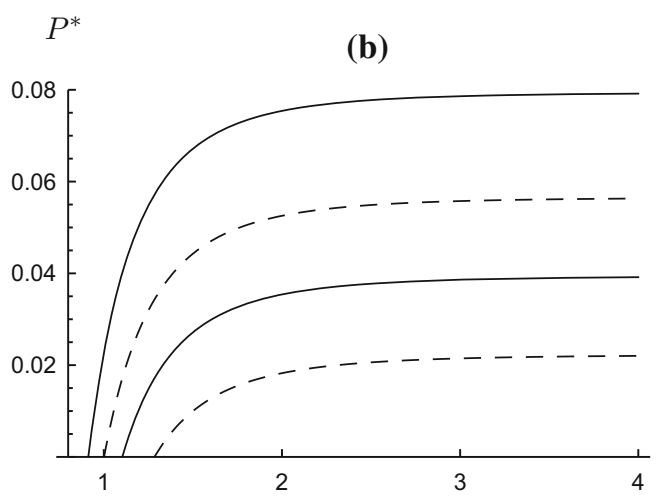

(d)

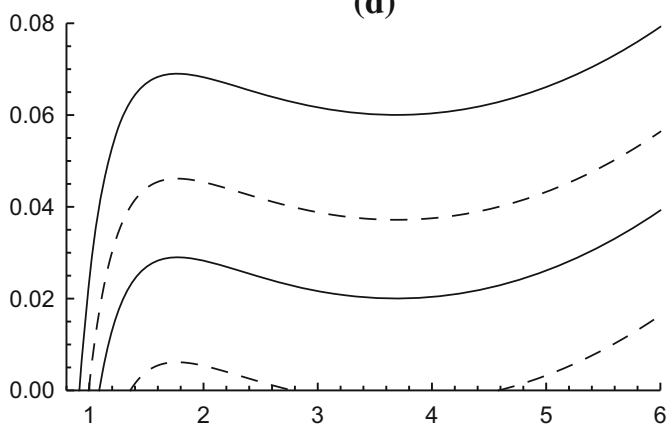

(f)

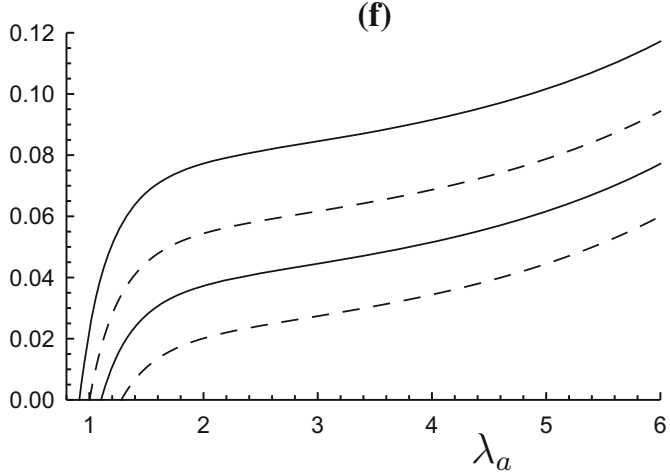

FIG. 1. Plots of the dimensionless pressure $P^{*}$ versus $\lambda_{a}$ with $\eta=1.1$ and $\lambda_{z}=1.2$ based on Eqs. (45) and (54) for the neoHookean, Ogden and Gent models in the first, second and third rows, respectively, and for fixed charges with $q^{*}=q / \mu \varepsilon=$ $0,1,5,10$ in the left-hand column and fixed potential difference in the right-hand column with $e^{*}=\varepsilon E_{0}^{2} / \mu=0,0.2,0.35,0.55$ in (d) and $e^{*}=\varepsilon E_{0}^{2} / \mu=0,0.2,0.35,0.5$ in (b) and (f). In each panel the value of $P^{*}$ decreases as the magnitude of the field measure increases

For the Gent model the results shown in Fig. 1e, f follow a similar trend to those in Fig. 1c, d except that the pressure increases monotonically with $\lambda_{a}$ up to a limiting value of $\lambda_{a}$ beyond the values shown in Fig. 1e, $\mathrm{f}$ for which $P^{*} \rightarrow \infty$, which is associated with so-called limiting chain extensibility.

In general, as can be seen from all the plots in Fig. 1, when an electric field is operative between the electrodes a lower pressure is required to obtain a prescribed circumferential stretch compared with that required in the purely elastic case. 
$F^{*}$

(a)

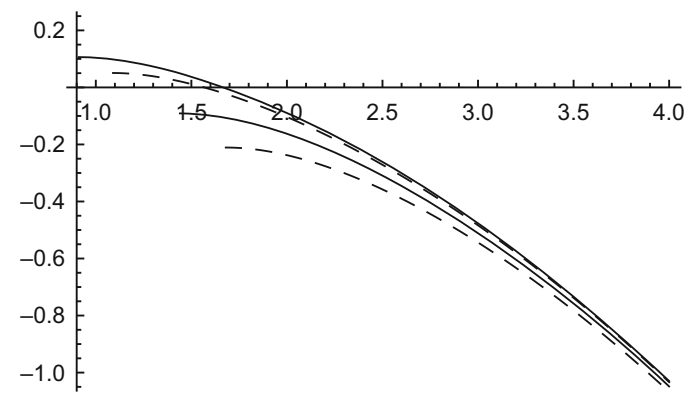

(c)

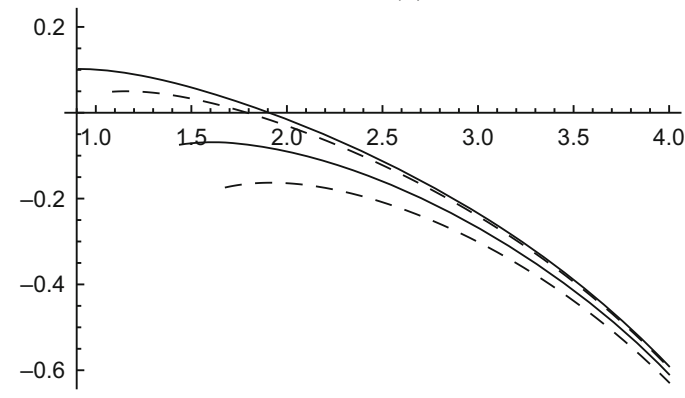

(e)

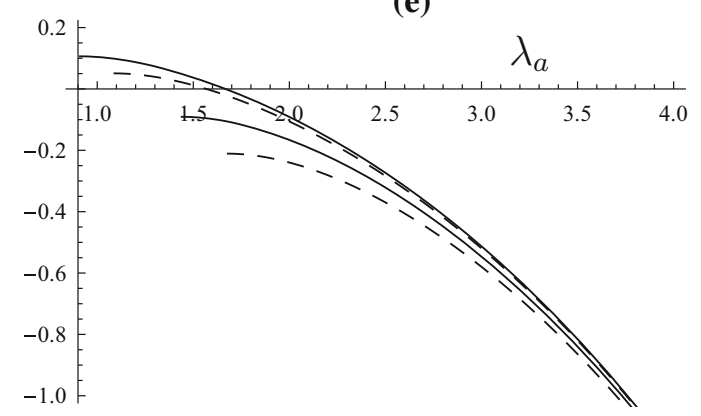

$$
F^{*}
$$

(b)

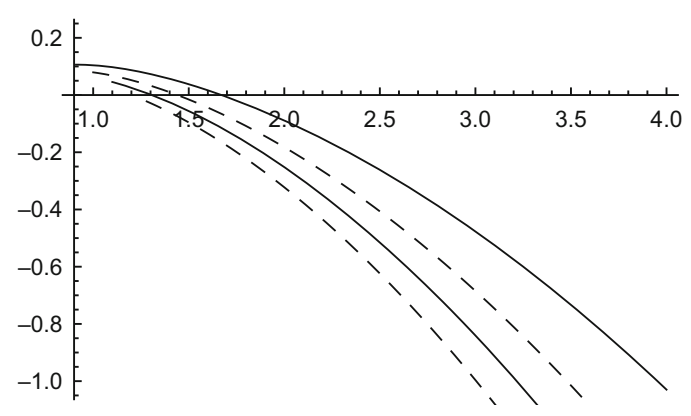

(d)

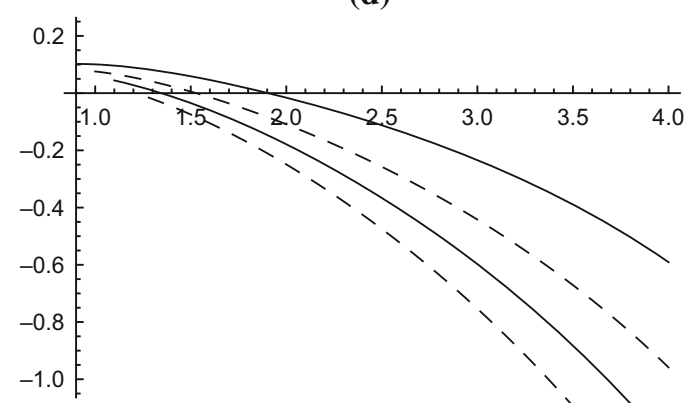

(f)

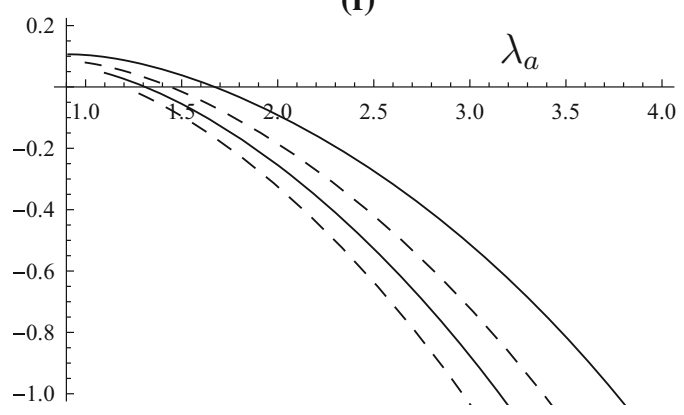

FIG. 2. Plots of non-dimensional reduced axial load $F^{*}$ versus $\lambda_{a}$ with $\eta=1.1$ and $\lambda_{z}=1.2$ based on Eqs. (46) and (55) for the neo-Hookean, Ogden and Gent models in the first, second and third rows, respectively, and for fixed charges with $q^{*}=0,1,5,10$ in the left-hand column and fixed potential difference with $e^{*}=0,0.2,0.35,0.5$ in the right-hand column. In each panel the value of $F^{*}$ decreases as the magnitude of the field measure increases

The plots in Fig. 1 are for $\lambda_{z}=1.2$. The plots are qualitatively similar for other values of $\lambda_{z}>1$ provided they are not unrealistically large. Values less than 1 can be associated with buckling instabilities in the absence of pressure or an electric field and are not considered here.

In Fig. 2, again with $\eta=1.1$ and $\lambda_{z}=1.2$, the effect of inflation on the reduced axial load is illustrated for the same material models as in Fig. 1 with the same layout of the six panels. The same trend can be observed for each of the material models in Fig. 2a-f, so we focus on the description of this trend without reference to a specific panel. The uppermost curve corresponds to the purely elastic case, which starts where $\lambda_{a}=\lambda_{z}^{-1 / 2}$ for $\lambda_{z}=1.2$. This requires a positive value of the reduced axial load. As inflation proceeds this positive value is maintained initially but then, after a certain value of $\lambda_{a}$, the axial load 
becomes negative, indicating that in its absence the tube would increase its length. An applied electric field causes an increase in $\lambda_{a}$ at zero pressure so that the corresponding curves for different values of $q^{*}$ or $e^{*}$ begin at the appropriate higher value of $\lambda_{a}$ and generally the value of $F^{*}$ decreases as the pressure increases. Thus, in general, an applied electric field counteracts the effect of the initial positive reduced axial load and tends to increase the length of the tube, thus requiring a negative reduced axial load to maintain the stretch $\lambda_{z}=1.2$ when the electric field strength becomes large enough. Fig. 2c is slightly different from the other panels in that, as $q^{*}$ increases, $F^{*}$ exhibits a maximum as the pressure increases, but then decreases monotonically, as in the other panels.

In Figs. 3 and 4 the counterparts of the results shown in Figs. 1 and 2 are illustrated for a tube with a thicker wall with $\eta=1.5$ and with the same values of $q^{*}$ and $e^{*}$, except that in Fig. $3 \mathrm{~d}$ the value $e^{*}=0.5$ is used instead of $e^{*}=0.55$ in Fig. $1 \mathrm{~d}$ so as to avoid the curve intersecting the $P^{*}=0$ axis. Qualitatively the plots are identical to those in Figs. 1 and 2, the main difference quantitatively being that the values of $P^{*}$ and $F^{*}$ in Figs. 3 and 4 are significantly larger than those in Figs. 1 and 2 for each $\lambda_{a}$ (by a factor of between about 4 and 6). Note that because of the neglect of end conditions and the assumed maintenance of the circular cylindrical shape the length of the tube does not feature in the calculations (except indirectly through $q$ ). For a thick-walled tube it can be expected, however, that shear tractions will be required on the cylinder ends in order to maintain the geometry. These are not considered here because our main interest is in relatively thin-walled tubes.

Our calculations show that the qualitative nature of the results remains the same as the wall thickness increases, except that the maximum/minimum for the Ogden model is removed for sufficiently large values of $\eta$, which are not our concern here. This suggests that the main features of interest can be captured by considering a thin-walled tube, and the thin-walled tube approximation is therefore examined in the following section.

\section{The thin-walled tube approximation}

For a thin-walled tube the general formulas (39) and (42) for $P$ and $F$ can be made explicit without specializing the form of energy function. For this purpose the small dimensionless parameter $\delta$ defined by $\delta=(B-A) / A$ is introduced. Then, from the expression $b^{2}=a^{2}+\lambda_{z}^{-1}\left(B^{2}-A^{2}\right)$ the approximation

$$
b \simeq a+\delta \lambda_{z}^{-1} \lambda_{a}^{-2} a
$$

is obtained to the first order in $\delta$.

The expression (39) is then approximated to the same order as

$$
P \simeq \frac{b-a}{a} \lambda_{a} \omega_{\lambda}^{*}\left(\lambda_{a}, \lambda_{z}, I_{4 a}\right)
$$

where $I_{4 a}$ is the value of $I_{4}$ on $r=a$, which is given by

$$
I_{4 a}=q .
$$

Thus,

$$
P \simeq \delta \lambda_{z}^{-1} \lambda_{a}^{-1} \omega_{\lambda}^{*}\left(\lambda_{a}, \lambda_{z}, q\right)
$$

to the first order in $\delta$.

The corresponding approximation for $F$ is obtained from (42) as

$$
F \simeq \delta \pi A^{2}\left[2 \omega_{\lambda_{z}}^{*}\left(\lambda_{a}, \lambda_{z}, q\right)-\lambda_{a} \lambda_{z}^{-1} \omega_{\lambda}^{*}\left(\lambda_{a}, \lambda_{z}, q\right)\right] .
$$

For the reduced energy function (43) with a general elastic term we can rewrite (71) as

$$
P \simeq \delta \lambda_{a}^{-1} \lambda_{z}^{-1}\left[\omega_{\lambda}\left(\lambda_{a}, \lambda_{z}\right)-\varepsilon^{-1} \lambda_{a}^{-3} \lambda_{z}^{-2} q\right] .
$$



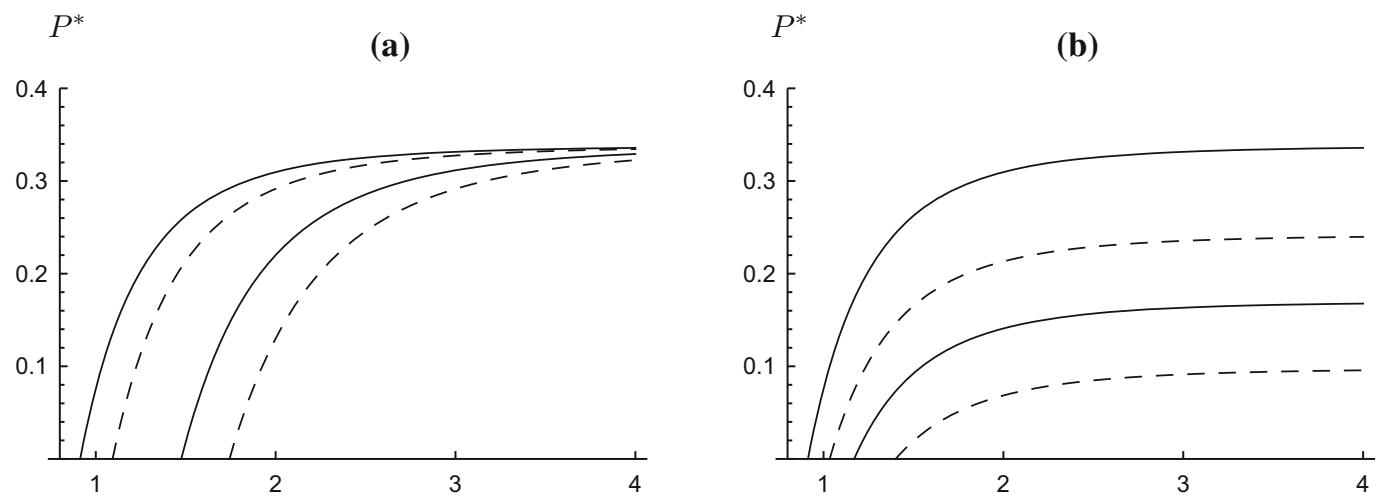

(c)
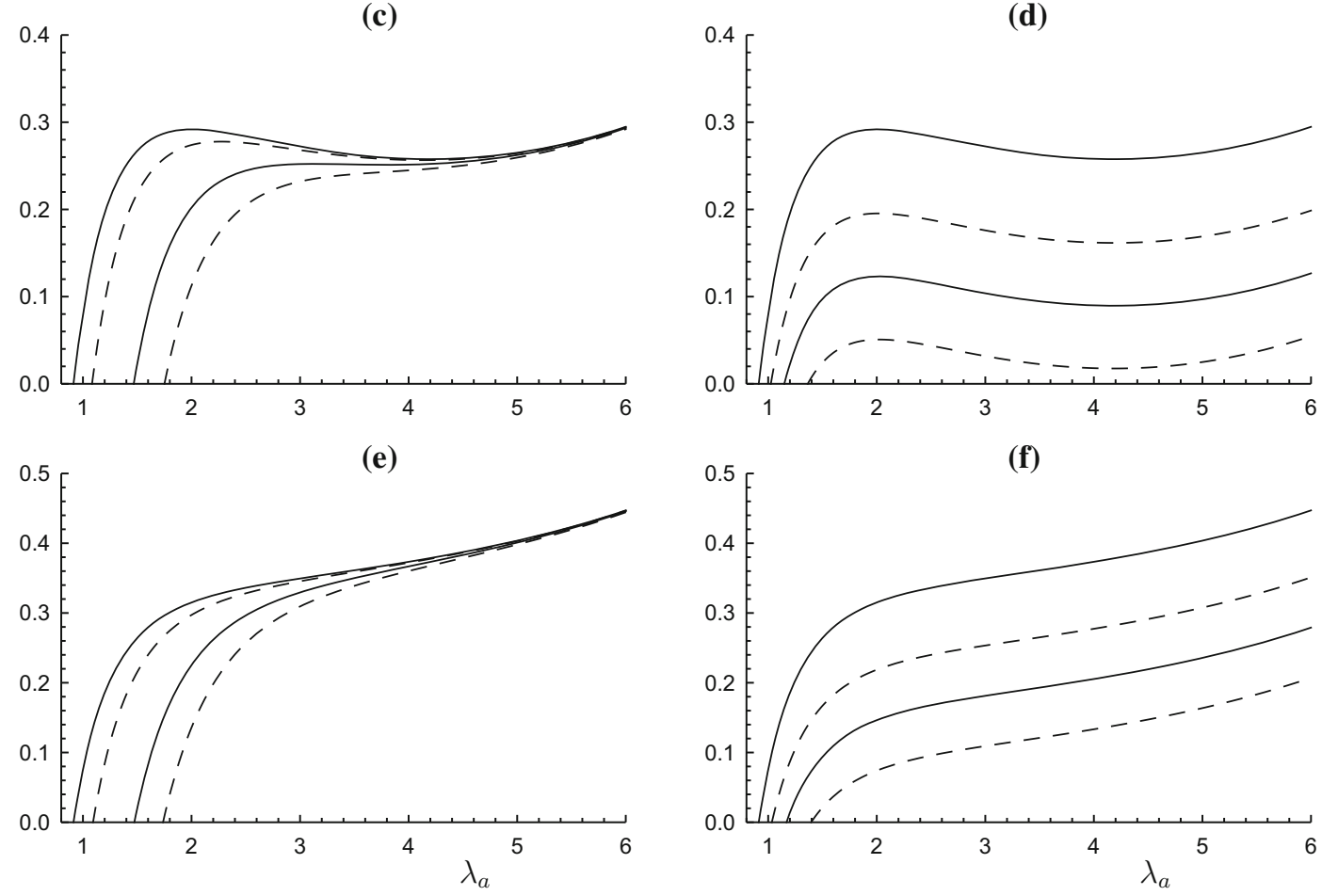

FIG. 3. Plots of the dimensionless pressure $P^{*}$ versus $\lambda_{a}$ with $\eta=1.5$ and $\lambda_{z}=1.2$ based on Eqs. (45) and (54) for the neoHookean, Ogden and Gent models in the first, second and third rows, respectively, and for fixed charges with $q^{*}=0,1,5,10$ in the left-hand column and fixed potential difference in the right-hand column with $e^{*}=0,0.2,0.35,0.5$. In each panel the value of $P^{*}$ decreases as the magnitude of the field measure increases

Clearly the influence of the charge (via $q$ ) on the pressure $P$ becomes less and less with increasing azimuthal stretch $\lambda_{a}$ at fixed $\lambda_{z}$.

Similarly, for the reduced energy function, the approximation for $F$ can be written

$$
F \simeq \delta \pi A^{2}\left[2 \omega_{\lambda_{z}}\left(\lambda_{a}, \lambda_{z}\right)-\lambda_{a} \lambda_{z}^{-1} \omega_{\lambda}\left(\lambda_{a}, \lambda_{z}\right)-\varepsilon^{-1} \lambda_{a}^{-2} \lambda_{z}^{-3} q\right] .
$$

Thus, as for the pressure, with increasing circumferential stretch $\lambda_{a}$ the influence of the electric field expressed in terms of the charge $q$ becomes less and less. 


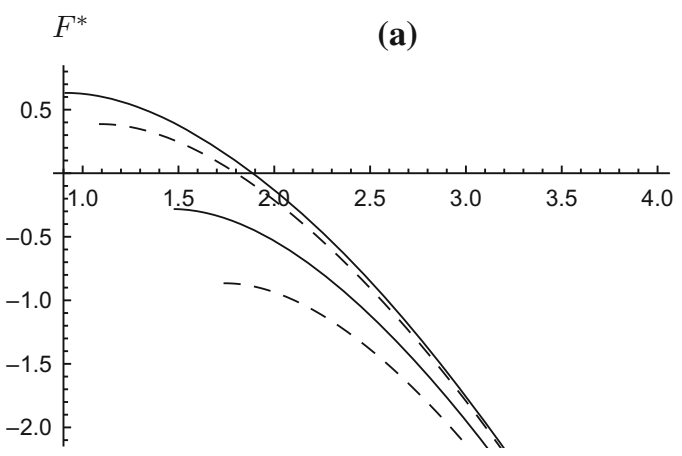

(c)

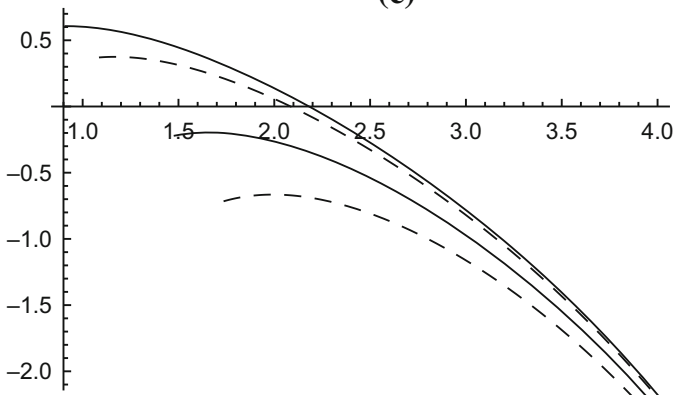

(e)

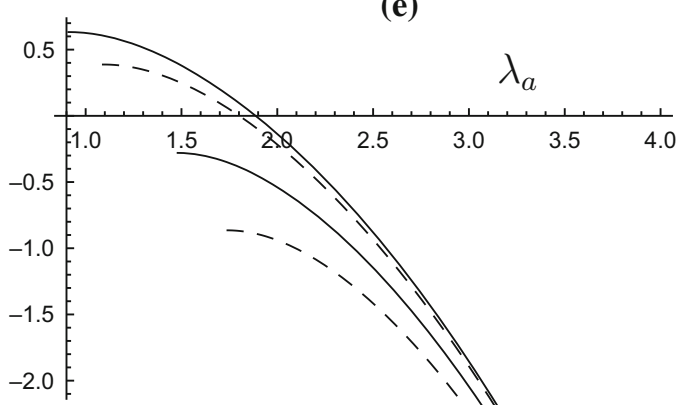

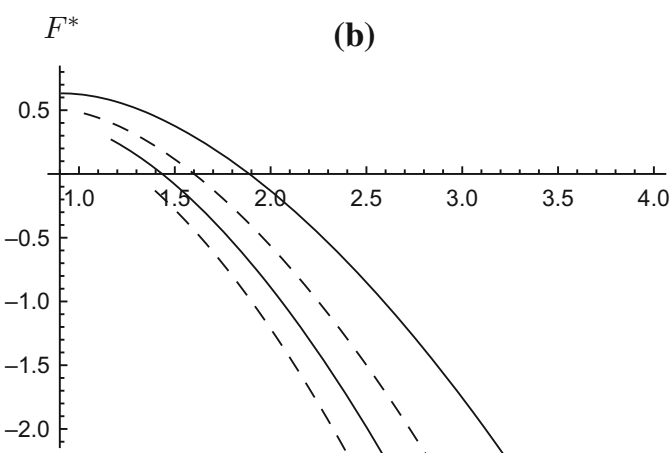

(d)

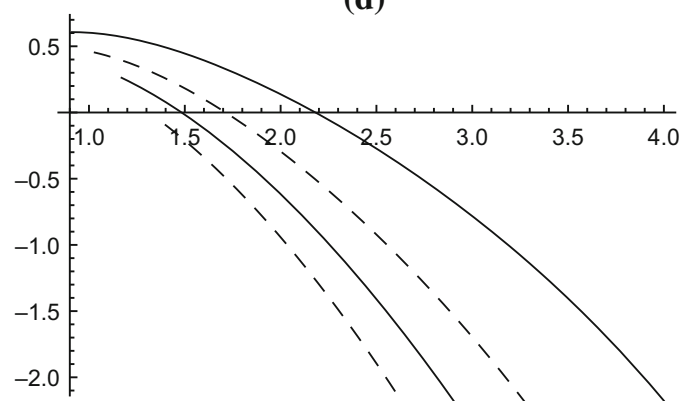

(f)

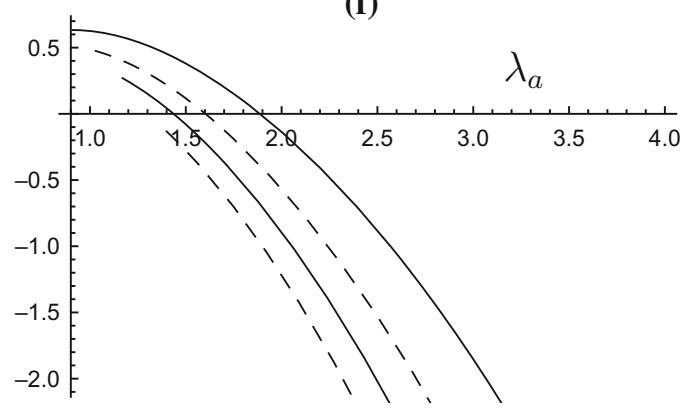

FIG. 4. Plots of non-dimensional reduced axial load $F^{*}$ versus $\lambda_{a}$ with $\eta=1.5$ and $\lambda_{z}=1.2$ based on Eqs. (46) and (55) for the neo-Hookean, Ogden and Gent models in the first, second and third rows, respectively, and for fixed charges with $q^{*}=0,1,5,10$ in the left-hand column and fixed potential difference with $e^{*}=0,0.2,0.35,0.5$ in the right-hand column. In each panel the value of $F^{*}$ decreases as the magnitude of the field measure increases

To the same approximation the connection (53) yields

$$
\varepsilon E_{0}^{2}=\frac{q}{\varepsilon \lambda_{z}^{4} \lambda_{a}^{4}},
$$

and (73) and (74) can be written in terms of $E_{0}$ as

$$
P \simeq \delta \lambda_{a}^{-1} \lambda_{z}^{-1}\left[\omega_{\lambda}\left(\lambda_{a}, \lambda_{z}\right)-\varepsilon \lambda_{a} \lambda_{z}^{2} E_{0}^{2}\right]
$$

and

$$
F \simeq \delta \pi A^{2}\left[2 \omega_{\lambda_{z}}\left(\lambda_{a}, \lambda_{z}\right)-\lambda_{a} \lambda_{z}^{-1} \omega_{\lambda}\left(\lambda_{a}, \lambda_{z}\right)-\varepsilon \lambda_{a}^{2} \lambda_{z} E_{0}^{2}\right]
$$


Thus, for a given potential difference the term in (76) involving $E_{0}$ is not affected by the azimuthal stretch $\lambda_{a}$. Therefore, in this case, the effect of an electric field is uncoupled from the stretch $\lambda_{a}$ for any fixed $\lambda_{z}$. On the other hand, the reduced axial load will be affected significantly by increasing circumferential stretch $\lambda_{a}$ for given nonzero $E_{0}$.

As far as the elastic contribution to the energy is concerned it is not necessary to restrict attention to isotropic materials. For example, the theory can accommodate fibre reinforcement with symmetrically arranged fibres, an arrangement commonly adopted for arterial wall tissue (see, for example, [22]), in which case $\omega\left(\lambda, \lambda_{z}\right)$ is not in general a symmetric function of $\lambda$ and $\lambda_{z}$ in contrast to the isotropic case. Otherwise the equations are unchanged. To include inextensible fibres, as in [13], an additional constraint is required.

\section{A note on activation}

We now express the formulas for $P$ and $F$ from Sect. 5 in the dimensionless forms

$$
P^{*}=\lambda_{a}^{-1} \lambda_{z}^{-1} \bar{\omega}_{\lambda}\left(\lambda_{a}, \lambda_{z}\right)-\lambda_{a}^{-4} \lambda_{z}^{-3} q^{*}, \quad F^{*}=2 \bar{\omega}_{\lambda_{z}}\left(\lambda_{a}, \lambda_{z}\right)-\lambda_{a} \lambda_{z}^{-1} \bar{\omega}_{\lambda}\left(\lambda_{a}, \lambda_{z}\right)-\lambda_{a}^{-2} \lambda_{z}^{-3} q^{*},
$$

from (73) and (74), and

$$
P^{*}=\lambda_{a}^{-1} \lambda_{z}^{-1} \bar{\omega}_{\lambda}\left(\lambda_{a}, \lambda_{z}\right)-\lambda_{z} e^{*}, \quad F^{*}=2 \bar{\omega}_{\lambda_{z}}\left(\lambda_{a}, \lambda_{z}\right)-\lambda_{a} \lambda_{z}^{-1} \bar{\omega}_{\lambda}\left(\lambda_{a}, \lambda_{z}\right)-\lambda_{a}^{2} \lambda_{z} e^{*},
$$

from (76) and (77), where $q^{*}=q /(\mu \varepsilon)$ and $e^{*}=\varepsilon E_{0}^{2} / \mu$, as used in Sect. $4, \bar{\omega}=\omega / \mu$, and $P^{*}=P /(\delta \mu)$ and $F^{*}=F /\left(\delta \mu \pi A^{2}\right)$, the latter two non-dimensionalizations being different from those used in Sect. 4 .

From either of $(78)$ or (79) it follows that

$$
F^{*}-\lambda_{a}^{2} P^{*}=2 \bar{\omega}_{\lambda_{z}}\left(\lambda_{a}, \lambda_{z}\right)-2 \lambda_{a} \lambda_{z}^{-1} \bar{\omega}_{\lambda}\left(\lambda_{a}, \lambda_{z}\right) .
$$

If there is no internal pressure $\left(P^{*}=0\right)$ then for a given (fixed) axial load $F^{*}$ this determines a connection between $\lambda_{a}$ and $\lambda_{z}$ (in general implicit), and, for an applied voltage (in terms of $e^{*}$ ) for example, Eq. (79) 1 provides a connection between $\lambda_{z}$ and $e^{*}$, i.e., it determines the change in $\lambda_{z}$ due to activation from its initial value at $e^{*}=0$. Similarly, if $F^{*}=0$ and $P^{*}$ is fixed activation with $e^{*}$ causes a change in $\lambda_{z}$.

For simplicity these general principles are now illustrated in respect of the neo-Hookean elasticity model (63), for which

$$
F^{*}-\lambda_{a}^{2} P^{*}=2\left(\lambda_{z}-\lambda_{a}^{2} \lambda_{z}^{-1}\right)
$$

For $P^{*}=0$ we then have

$$
\lambda_{a}^{2} \lambda_{z}=\left(1-\lambda_{z}^{2} e^{*}\right)^{-1 / 2},
$$

which requires that $\lambda_{z}^{2} e^{*}<1$. Note, in particular, that in the limit $\lambda_{z}^{2} e^{*} \rightarrow 1, \lambda_{a} \rightarrow \infty$ and the wall thickness decreases to zero! Equation (81) requires that $\lambda_{z}>\lambda_{a}$ for $F^{*}>0$. From (81) it also follows that

$$
F^{*}=2 \lambda_{z}-2 \lambda_{z}^{-2}\left(1-\lambda_{z}^{2} e^{*}\right)^{-1 / 2} .
$$

For several fixed positive values of $F^{*}$ the interdependence of $e^{*}$ and $\lambda_{z}$ is illustrated in Fig. 5a. In terms of different variables similar plots were provided in [16] for different values of the initial axial stretch (equivalently, different values of $F^{*}$ ) and for a thick-walled tube with $B / A=2$. In [16] the maxima on the curves were interpreted as corresponding to loss of electromechanical stability.

For contrast we now consider activation at fixed pressure and zero axial load, so that, from (81),

$$
P^{*}=2 \lambda_{z}^{-1}-2 \lambda_{z} \lambda_{a}^{-2}
$$

which requires $\lambda_{a}>\lambda_{z}$ for $P^{*}>0$, while $F^{*}=0$ yields the quadratic

$$
\left(\lambda_{z}^{2} e^{*}+1\right) \lambda_{a}^{4}-2 \lambda_{z}^{2} \lambda_{a}^{2}+\lambda_{z}^{-2}=0
$$



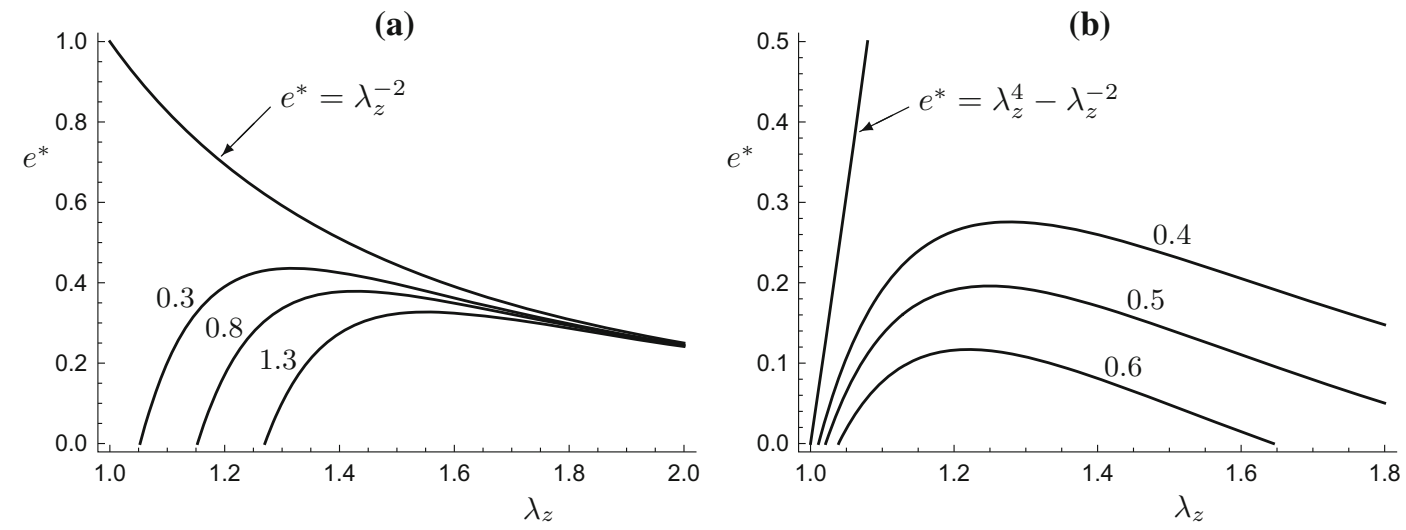

FIG. 5. a For $P^{*}=0$ : plots of the activation potential (as measured by $e^{*}$ ) versus the resulting axial stretch $\lambda_{z}$ for the indicated fixed values $0.3,0.8,1.3$ of the dimensionless axial load $F^{*}$ (corresponding to initial stretches, for $e^{*}=0$, of approximately $1.053,1.153,1.27$, respectively), together with the limiting curve defined by $\lambda_{z}^{2} e^{*}=1$. b For $F^{*}=0$ : plots of the activation potential (as measured by $e^{*}$ ) versus the resulting axial stretch $\lambda_{z}$ for the indicated fixed values $0.4,0.5,0.6$ of the dimensionless pressure $P^{*}$ (corresponding to initial stretches of approximately 1.01, 1.02, 1.04, respectively), together with the limiting curve defined by $e^{*}=\lambda_{z}^{4}-\lambda_{z}^{-2}$

for $\lambda_{a}^{2}$, the only solution of which consistent with $\lambda_{a}>\lambda_{z}$ being

$$
\lambda_{a}^{2}=\frac{\lambda_{z}^{2}+\sqrt{\lambda_{z}^{4}-\lambda_{z}^{-2}-e^{*}}}{\lambda_{z}^{2} e^{*}+1},
$$

which requires $\lambda_{z}^{4}-\lambda_{z}^{-2}>e^{*}$. Hence

$$
P^{*}=2 \lambda_{z}^{-1}-\frac{2 \lambda_{z}\left(\lambda_{z}^{2} e^{*}+1\right)}{\lambda_{z}^{2}+\sqrt{\lambda_{z}^{4}-\lambda_{z}^{-2}-e^{*}}},
$$

and this equation is the basis for the plots in Fig. 5b in which the interdependence of $e^{*}$ and $\lambda_{z}$ is illustrated for several fixed values of $P^{*}$.

As for the case with $P^{*}=0$ and fixed $F^{*}$ there is a maximum actuation voltage for each considered value of $P^{*}$ and again the maxima are associated with loss of electromechanical stability. However, for the considered neo-Hookean model in the absence of a voltage the radius can increase indefinitely as the pressure approaches a finite asymptote, and this behaviour is a reflection of the limited applicability of the neo-Hookean model, which is only realistic for stretches up to about 2 . This should be borne in mind when assessing the results of activation. For models such as those in [18] and [23] that are valid for a wider range of deformations than for the neo-Hookean model there is no theoretical limit to the allowable voltage, which can increase indefinitely with the axial stretch, possibly with an intermediate maximum followed by a minimum, as is the case for a particular Arruda-Boyce model considered in [16].

Next, based on equations in (78), we consider activation with specified charge rather than a potential, in which case, with $P^{*}=0$ we obtain

$$
\lambda_{a}^{2} \lambda_{z}=\sqrt{1+q^{*}}
$$

and

$$
F^{*}=2 \lambda_{z}-2 \lambda_{z}^{-2} \sqrt{1+q^{*}} .
$$

For $F^{*}=0$, on the other hand, we have

$$
\lambda_{a}^{2} \lambda_{z}=\lambda_{z}^{3}+\sqrt{\lambda_{z}^{6}-1-q^{*}}
$$



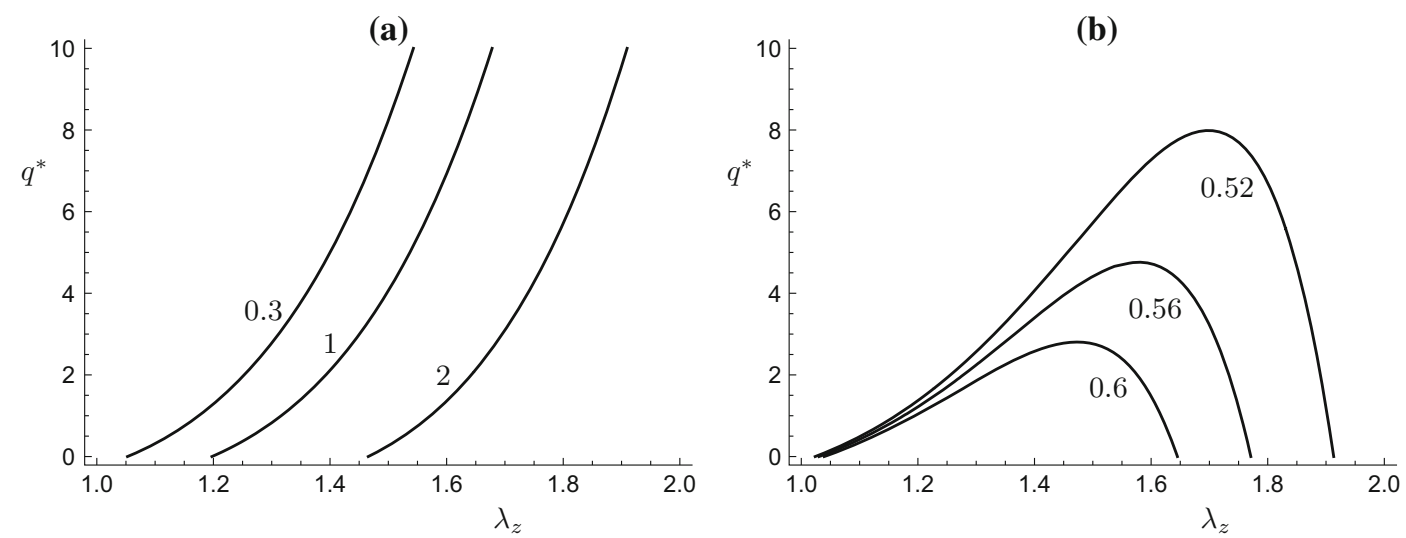

FIG. 6. a For $P^{*}=0$ : plots of the activation charge (as measured by $q^{*}$ ) versus the resulting axial stretch $\lambda_{z}$ for the indicated fixed values $0.3,1,2$ of the dimensionless axial load $F^{*}$ (corresponding to initial stretches, for $q^{*}=0$, of approximately $1.05,1.2,1.47$, respectively). b For $F^{*}=0$ : plots of the activation charge (as measured by $q^{*}$ ) versus the resulting axial stretch $\lambda_{z}$ for the indicated fixed values $0.52,0.56,0.6$ of the dimensionless pressure $P^{*}$ (corresponding to initial stretches of approximately 1.02, 1.03, 1.04, respectively)

and

$$
P^{*}=2 \lambda_{z}^{-1}-\frac{2 \lambda_{z}^{2}}{\lambda_{z}^{3}+\sqrt{\lambda_{z}^{6}-1-q^{*}}} .
$$

Results for $P^{*}=0$ and $F^{*}=0$, respectively, are illustrated in Fig. 6a, b with $q^{*}$ plotted against $\lambda_{z}$ analogously to those in Fig. 5a, b for $e^{*}$ against $\lambda_{z}$. In Fig. 6a the plots are for $F^{*}=0,0.3,1,2$ and in Fig. $6 \mathrm{~b}$ for $P^{*}=0.52,0.56,6$. In Fig. 6a, in contrast to Fig. 5a, there is no maximum and the stretch $\lambda_{z}$ increases monotonically with the applied charge, whereas in Fig. $6 \mathrm{~b}$ there is a maximum for any pressure below the maximum attainable $\left(P^{*} \simeq 0.75\right)$ with $q^{*}=0$ for the neo-Hookean material and this has a similar 'instability' interpretation as for a fixed $F^{*}$ at $P^{*}=0$ in Fig. 5.

\section{Concluding remarks}

In this paper the general formulation of nonlinear isotropic electroelasticity in the form developed by Dorfmann and Ogden [6] has been applied to the prototype problem of a circular cylindrical tube of dielectric elastomer with compliant electrodes on its major surfaces. Without specialization of the constitutive law general expressions have been obtained for the internal pressure in the tube and axial load on its ends when subject to a radial electric field generated by a potential difference between the electrodes while the circular cylindrical geometry is maintained. The general results are then applied to a material model for which the electrostatic part of the constitutive law is linear with a deformation independent permittivity, and the electroelastic response of the tube has been illustrated for three different models of the elastic contribution to the constitutive law from rubber elasticity.

It is, of course, a simplifying assumption that the permittivity of the material is independent of the deformation, an assumption that runs counter to experimental evidence, at least for some dielectric elastomers. For example, in an extensive series of experiments on the acrylic elastomer VBH 4910 Wissler and Mazza [24] showed that the permittivity decreases with stretching, and this should be taken into account in the modelling in situations where the deformations are relatively large. Such an influence is easily accommodated within the general constitutive framework presented in Sect. 2.4 and its specialization to the considered geometry in Sect. 3. However, in general this leads to a more complicated analysis 
and numerical solution will for the most part be required. Specific models which do include deformation dependent permittivity have been examined in a variety of boundary-value problems by Dorfmann and Ogden [6,7,25-27] and [8], while the influence of deformation dependent permittivity on stability considerations has been addressed in $[28,29]$ and [30].

To incorporate a fibre structure within the constitutive law is feasible but requires a more involved theory with a much larger set of invariants than those considered here in general, as exemplified in the case of a transversely isotropic electroelastic material by Bustamante [31].

\section{Acknowledgments}

The work of Andrey Melnikov was funded by a University of Glasgow studentship.

Open Access. This article is distributed under the terms of the Creative Commons Attribution 4.0 International License (http://creativecommons.org/licenses/by/4.0/), which permits unrestricted use, distribution, and reproduction in any medium, provided you give appropriate credit to the original author(s) and the source, provide a link to the Creative Commons license, and indicate if changes were made.

\section{References}

[1] Toupin, R.A.: The elastic dielectric. J. Ration. Mech. Anal. 5, 849-915 (1956)

[2] Hutter, K., van de Ven, A.A.F.: Field matter interactions in thermoelastic solids. Lecture notes in physics vol. 88. Springer, Berlin (1978)

[3] Nelson, D.F.: Electric, Optic, and Acoustic Interactions in Dielectrics. Wiley, New York (1979)

[4] Maugin, G.A.: Continuum Mechanics of Electromagnetic Solids. North Holland, Amsterdam (1988)

[5] Eringen, A.C., Maugin, G.A.: Electrodynamics of Continua, vol. I. Springer, New York (1990)

[6] Dorfmann, A., Ogden, R.W.: Nonlinear electroelasticity. Acta Mech. 174, 167-183 (2005)

[7] Dorfmann, A., Ogden, R.W.: Nonlinear electroelastic deformations. J. Elast. 82, 99-127 (2006)

[8] Dorfmann, A., Ogden, R.W.: Nonlinear Theory of Electroelastic and Magnetoelastic Interactions. Springer, New York (2014)

[9] Pelrine, R.E., Kornbluh, R.D., Joseph, J.P.: Electrostriction of polymer dielectrics with compliant electrodes as a means of actuation. Sens. Actuators A Phys. 64, 77-85 (1998)

[10] Pelrine, R., Sommer-Larsen, P., Kornbluh, R., Heydt, R., Kofod, G., Pei, Q., Gravesen, P.: Applications of dielectric elastomer actuators. In: Bar-Cohen, Y. (ed.) Smart Structures and Materials 2001, Electroactive Polymer Actuators and Devices, Proceedings of SPIE, vol. 4329. SPIE, Bellingham, WA (2001)

[11] Dorfmann, A., Ogden, R.W.: Nonlinear response of an electroelastic spherical shell. Int. J. Eng. Sci. 85, 163-174 (2014)

[12] Reynolds, D.B., Repperger, D.W., Phillips, C.A., Bandry, G.: Modeling the dynamic characteristics of pneumatic muscle. Ann. Biomed. Eng. 31, 310-317 (2003)

[13] Goulbourne, N.C.: A mathematical model for cylindrical, fiber reinforced electro-pneumatic actuators. Int. J. Solids Struct. 46, 1043-1052 (2009)

[14] Carpi, F., De Rossi, D., Kornbluh, R., Pelrine, R., Sommer-Larsen, P.: Dielectric Elastomers as Electromechanical Transducers. Elsevier, Amsterdam (2008)

[15] Carpi, F., De Rossi, D.: Dielectric elastomer cylindrical actuators: electromechanical modelling and experimental evaluation. Mat. Sci. Eng. C Biomim. Supramol. Syst. 24, 555-562 (2004)

[16] Zhu, J., Stoyanov, H., Kofod, G., Suo, Z.: Large deformation and electromechanical instability of a dielectric elastomer tube actuator. J. Appl. Phys. 108, 074113 (2010)

[17] Zhao, X., Hong, W., Suo, Z.: Electromechanical hysteresis and coexistent states in dielectric elastomers. Phys. Rev. B 76, $134113(2007)$

[18] Ogden, R.W.: Large deformation isotropic elasticity — on thecorrelation of theory and experiment for incompressible rubberlike solids. Proc. R. Soc. Lond. A 326, 565-584 (1972)

[19] Gent, A.N.: A new constitutive relation for rubber. Rubber Chem. Technol. 69, 59-61 (1996)

[20] Wolfram Research, Inc., Mathematica, Version 10.4, Champaign (2016)

[21] Rudykh, S., Bhattacharya, K., deBotton, G.: Snap-through actuation of thick-wall electroactive balloons. Int. J. NonLin. Mech. 47, 206-209 (2012)

[22] Holzapfel, G.A., Ogden, R.W.: Constitutive modelling of arteries. Proc. R. Soc. Lond. A 466, 1551-1596 (2010) 
[23] Arruda, E.M., Boyce, M.C.: A three-dimensional model for the large stretch behavior of rubber elastic materials. J. Mech. Phys. Solids 41, 389-412 (1993)

[24] Wissler, M., Mazza, E.: Electromechanical coupling in dielectric elastomer actuators. Sens. Actuators A 138, 384-393 (2007)

[25] Dorfmann, A., Ogden, R.W.: Nonlinear electroelasticity: incremental equations and stability. Int. J. Eng. Sci. 48, 1-14 (2010)

[26] Dorfmann, A., Ogden, R.W.: Electroelastic waves in a finitely deformed electroactive material. IMA J. Appl. Math. 75, 603-636 (2010)

[27] Dorfmann, L., Ogden, R.W.: Instabilities of an electroelastic plate. Int. J. Eng. Sci. 77, 79-101 (2014)

[28] Zhao, X., Suo, Z.: Electrostriction in elastic dielectrics undergoing large deformation. J. Appl. Phys. 104, 123530 (2008)

[29] Liu, Y., Liu, L., Sun, S., Leng, J.: Electromechanical stability of a Mooney-Rivlin-type dielectric elastomer with nonlinear variable permittivity. Polym. Int. 59, 371-377 (2010)

[30] Jimé nez, S.M.A., McMeeking, R.M.: Deformation dependent dielectric permittivity and its effect on actuator performance and stability. Int. J. Non-Lin. Mech 57, 183-191 (2013)

[31] Bustamante, R.: Transversely isotropic non-linear electro-active elastomer. Acta Mech. 206(2009), 237-259 (2009)

Andrey Melnikov and Ray W. Ogden

School of Mathematics and Statistics

University of Glasgow

Glasgow

UK

e-mail: rwo@maths.gla.ac.uk

(Received: September 27, 2016) 